Gerión. Revista de Historia Antigua

ISSN: 0213-0181

http://dx.doi.org/10.5209/geri.71951

\title{
Dos inscripciones tardoantiguas donadas al Museo Arqueológico Nacional (Madrid) ${ }^{1}$
}

Javier Santiago Fernández²

Recibido: 28 de octubre de 2019 / Aceptado: 22 de junio de 2020

Resumen. El presente artículo estudia dos inscripciones del Museo Arqueológico Nacional. Se analizan tanto las propias inscripciones como la documentación sobre ellas existente en el archivo del Museo. En ambas se ofrece un estudio integral, tanto de los elementos externos como internos, analizando la escritura y los formulismos utilizados. Una de ellas había sido publicada previamente; aquí se ofrece una propuesta razonada de datación y se ofrecen todos los datos conocidos sobre su hallazgo y entrada en el Museo. La otra es un fragmento inédito.

Palabras clave: epigrafía; tardoantigüedad; coleccionismo.

\section{[en] Two Late Antique Inscriptions Donated to Museo Arqueológico Nacional (Madrid)}

Abstract. This article studies two inscriptions which are preserved in Museo Arqueológico Nacional (Madrid). We have analysed the epigraphs and all the documents stored in the Museum's archive which contain information about these inscriptions. The method of researching studies external and internal elements, analysing the writing and the formulae that are used in the texts. One of them had been published previously; we suggest a reasoned proposal for dating and we give all the known information about its archeological finding and its entrance in the Museum. The other is an unpublished fragment. Keywords: Epigraphy; Late-Antiquity; Collectionism.

Sumario: 1. Introducción. 2. Epitaphium sepulchrale de Firmana. 3. Epitaphium sepulchrale anónimo. 4. A modo de conclusión. 5. Referencias bibliográficas.

Cómo citar: Santiago Fernández, J. (2020): Dos inscripciones tardoantiguas donadas al Museo Arqueológico Nacional (Madrid), en Gerión 38/2, 623-638.

1 Este trabajo está adscrito al proyecto de investigación Redes AVIPES-CM (ref. H2019-HUM/5742), de la Comunidad de Madrid/Fondo Social Europeo.

2 Universidad Complutense de Madrid.

E-mail: santiago@ucm.es

ORCID: 0000-0001-6446-1963 


\section{Introducción}

Se presenta aquí un estudio de dos inscripciones conservadas en el Museo Arqueológico Nacional (Madrid), procedentes de sendas donaciones o ventas, cuya adquisición está documentada en los fondos del Archivo del Museo. Se trata de dos piezas insuficientemente estudiadas. El conocimiento actual de la primera de ellas se basa fundamentalmente en la aportación de Vives, ${ }^{3}$ cuyo texto y edición presenta algunos errores que han arrastrado investigadores posteriores. La segunda es un fragmento inédito. Así pues, pretendo ofrecer un estudio que aporte un pequeño grano al progreso del conocimiento epigráfico, pero también al de las propias piezas en cuanto objetos museológicos, vinculados con la historia del coleccionismo y del comercio de antigüedades.

\section{Epitaphium sepulchrale de Firmana (Fig. 1)}

Lo componen cuatro fragmentos de mármol blanco correspondientes a una placa. Procede de la necrópolis de Vega del Mar (San Pedro de Alcántara, Málaga). Tiene una anchura máxima de $43 \mathrm{~cm}$, una altura máxima de $28,50 \mathrm{~cm}$ y un grosor de 3,70 $\mathrm{cm}$. En la parte superior, partiendo el primer renglón, se inserta un crismón en el interior de una corona de laurel, cuyas dimensiones son 5,24 x 5,30 cm. Su número de inventario en el MAN es 57848.

FiRMANA IN(Chrismo)FAS AṆ[IMA]
DVLCIS VIXIT IN BONIS
ANNIS DVOBṾṢ MENSES
[O]CTO RECOLLECTA ẸST IN
[P]ACE SEPTIMỴ [K]ALEN
[DA]S FEBRVAṚ[IAS DI]ES SA
[TV]RNI +[---

Firmana infa $<n>s$, anima / dulcis, vixit in bonis $\beta$ annis duobus, menses / octo. Recollecta est in / pace septim ' ${ }^{\top}{ }^{7}$ kalen ${ }^{\circ}$ das februarias die $\{s\}$ Sa/turni [- - -].

La niña Firmana, de alma dulce, vivió entre los buenos durante dos años y ocho meses. Fue recogida en paz el día séptimo antes de las kalendas de febrero (25 de enero), el día de Saturno (sábado) ...

Bibliografía: ICERV 140; Taracena Aguirre 1947, 127; Rodríguez Oliva 1984, 462-463; García de Castro 1995-1996, 204; Del Hoyo Calleja 2016, 59.

lín. 1: ICERV, infas; Rodríguez Oliva, ANIMA; García de Castro, infas; Del Hoyo Calleja, infa(n)s. La no presencia de signo abreviativo y la ausencia de otras abreviaturas en el texto me lleva a desechar esta solución y a considerar la ausencia de la n simplemente resultado de una incorrección o de un olvido por parte del scriptor.

lín. 4: Rodríguez Oliva, OCTO.

lín. 5: ICERV, septimo; Rodríguez Oliva, PACE y SEPTIMO; García de Castro,

Vives 1969 (en adelante citado ICERV). 
septim[o]; Del Hoyo Calleja, septim[o]. En la inscripción es fácilmente perceptible la presencia de un trazo diagonal de izquierda a derecha que no puede obedecer a otra cosa que al primer trazo de la $\mathrm{V}$, incorrección que estaría en consonancia con otras constatadas.

lín. 6:ICERV, [d]as; Taracena, februarii[as]; Rodríguez Oliva, DASFREBVARIAS DIES; García de Castro, [d]as, [di]e; Del Hoyo Calleja, [d]as. De nuevo en esta línea observamos una incorrección, al declinar la palabra dies en nominativo, en lugar de en ablativo.

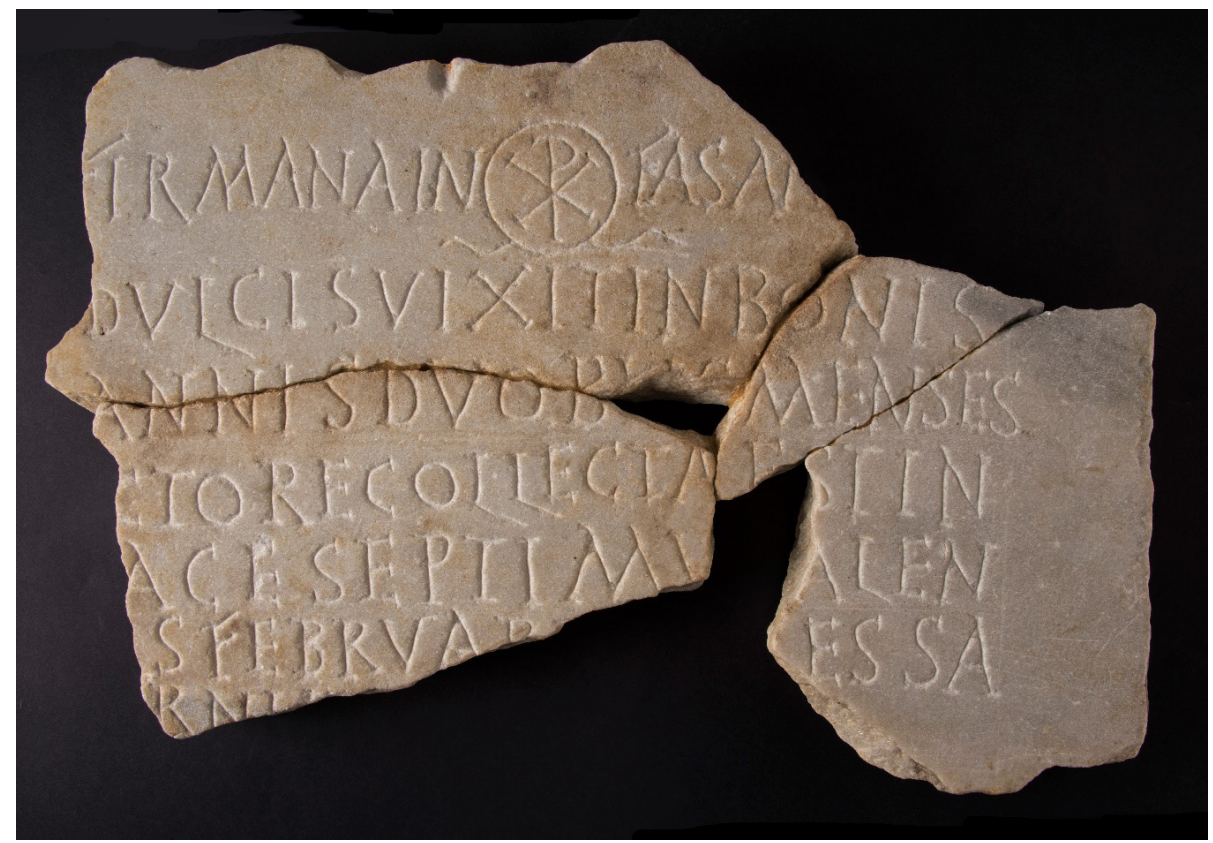

Fig. 1: Epitaphium sepulchrale de Firmana (C) Museo Arqueológico Nacional).

La restitución de lo que figuró después de Saturni es prácticamente imposible. Se observan aparentes restos de un trazo y podríamos pensar en el año de la Era como forma de poner la data completa después de indicar el día de la deposición o la muerte, hecho muy frecuente en la epigrafía cristiana hispana, especialmente en la Bética y en la Lusitania. Sin embargo, la antigüedad de la inscripción, que luego analizaré, lleva no solo a dudar de la aparente certeza de que el año de la Era tenga que figurar a continuación de la mención del día del óbito o la deposición, sino que creo que lo más probable es que no se consignase. Así parecen indicarlo numerosas inscripciones del siglo IV o primera mitad del V que no incluyen el año en la data. Buen ejemplo es el emeritense epitafio de Bonifatia, con una estructura formular similar al de Firmana, que ha de ser anterior al 440 por su formulario, según Joaquín $\mathrm{M}^{\mathrm{a}}$ de Navascués; ${ }^{4}$ vemos cómo después de consignar la fórmula notificativa, recepta in pace, escribe el día en que esto se produjo: diae II idus februarias. Caso similar es el epitafio de Flavius Salutius, procedente de Cabra (Córdoba), atribuido por Armin

Navascués 2019, 788. Editado también por ICERV 23 y Ramírez Sádaba - Mateos Cruz 2000, 62-63, nº 25 (en adelante $\mathrm{CICMe}$ ). 
Stylow al siglo IV. 5 Aunque es cierto que el primer epitafio datado es del 381, el desaparecido epitafio de Bracarius, con el año de la Era a continuación del día de fallecimiento, ${ }^{6}$ en la Bética no conocemos un epígrafe datado con seguridad, el de Licinia, ${ }^{7}$ procedente de Cazalla de la Sierra, hasta casi un siglo después, en concreto hasta el $485^{\circ}$. Creo que esta fecha en la Bética puede ratificar las dudas que la data tan temprana del epígrafe de Bracarius suscitó en Navascués, basándose en que es una inscripción desaparecida; ${ }^{9}$ de hecho, en Mérida, el siguiente epitafio datado es el de Octavia,${ }^{10} \mathrm{del} 442$, fecha que parece más plausible en relación con la introducción de la data por la Era en la Bética. Independientemente de esta última disquisición considero que la segunda mitad del siglo $\mathrm{V}$ es una fecha muy tardía para el epitafio de Firmana, por lo que, de acuerdo a los datos anteriores, creo que debemos rechazar la restitución a partir del año de la Era.

El origen del núcleo poblacional de la colonia de San Pedro de Alcántara se debe a la iniciativa de Manuel Gutiérrez de la Concha e Irigoyen, I marqués del Duero, quien vio las posibilidades agrícolas que ofrecía la zona para el cultivo de la caña de azúcar y en 1860 fundó la colonia. Posteriormente, en 1910, fue adquirida por la Sociedad General Azucarera de España. Las excavaciones arqueológicas se debieron a la iniciativa de José Martínez Oppelt, administrador de la colonia, ante la perspectiva que ofrecían los hallazgos casuales que se habían realizado gracias a los hoyos efectuados para la plantación de eucaliptos. La Junta Superior de Excavaciones y Antigüedades otorgó el preceptivo permiso de excavación por real orden de 7 de julio de $1916 .{ }^{11}$ Se trabajó durante los años 1915 y 1916, obteniendo resultados importantes, según indica José Pérez de Barradas. Entre los hallazgos se sitúa la inscripción que aquí analizamos. Un estudio inicial de ella fue realizado por Manuel Gómez-Moreno. ${ }^{12}$ La necrópolis fue situada por Pérez de Barradas entre los siglos IV y VII, en función de las monedas encontradas ${ }^{13}$ si bien la cronología de la inscripción puede ser precisada mucho más, según analizaré más adelante.

Los materiales descubiertos se mantuvieron en la finca hasta 1929, cuando fueron trasladados a Madrid y custodiados en la sede de la Sociedad Colonia San Pedro de Alcántara, ${ }^{14}$ entre ellos, sin duda, la inscripción objeto de nuestro interés. Posteriormente, esta pieza, y otros diferentes objetos arqueológicos, ingresaron en el Museo tras la donación efectuada por el Presidente del Consejo de Administración de la Compañía General Azucarera de España. La donación fue aceptada por el Patronato del Museo en su reunión de 11 de julio de 1941. En la lista de materiales recibidos la inscripción objeto de nuestro estudio figura con el número 79 y es sucintamente descrita como "Lápida con inscripción romano-cristiana en cuatro fragmentos". Más escueta, e incluso imprecisa, es aún la descripción realizada en la relación de objetos

\footnotetext{
$C I L \mathrm{II}^{2} / 5,334$.

CICMe 26.

ICERV 115.
}

Es anterior, del 466, otro procedente, si bien desaparecido, de Santa María de Regla (ICERV 141), del conventus gaditano, el mismo al que corresponde la inscripción de Firmana, pero el propio Vives ya calificó este texto de poco fiel y seguramente con correcciones ortográficas.

Navascués 2019, 779.

ICERV 24; CICMe 46.

Pérez de Barradas 1932, 54.

Pérez de Barradas 1930, 6.

Pérez de Barradas 1930, 8.

Pérez de Barradas 1932, 54. 
encontrados en la excavación: "Cuatro trozos de lápida con inscripción romana". Estas son las escasas noticias que sobre el origen de la inscripción nos proporciona el archivo del Museo. ${ }^{15}$

Esta documentación, además de los trabajos de Pérez de Barradas publicando los resultados de la excavación, nos permiten ver la aportación empresarial al desarrollo de la Arqueología y de la Epigrafía, primero fomentando la excavación y posteriormente donando al Museo Arqueológico Nacional importantes piezas, como esta que nos ocupa, que contribuyen al conocimiento arqueológico y epigráfico de nuestro país. No conocemos las razones concretas que impulsaron ni la excavación, ni la donación.

Entrando en el análisis de los elementos externos de la inscripción, la placa es uno de los soportes más frecuentes en las inscripciones cristianas, al ser idóneo para cerrar las fosas y lóculos de los enterramientos, además de ser relativamente asequible desde el punto de vista económico. El único elemento iconográfico de la placa es el crismón, el cristograma constantiniano que identifica universalmente el cristianismo desde la batalla del Puente Milvio en el $312^{16}$ y uno de los símbolos cristológicos más utilizados en los epígrafes cristianos; de hecho, es una de las más evidentes muestras de la existencia de una "cultura visual" 17 en los epitafios cristianos. Se sitúa en la parte superior, partiendo el primer renglón de escritura. Se trata de un crismón clásico, con la P cerrada, situado en el interior de una corona de laurel, de ejecución muy sencilla, tanto que se realiza mediante un simple círculo de cuya parte inferior salen dos trazos ondulados a modo de ínfulas. La unión del símbolo pagano de la corona de laurel, identificativo del triunfo, con el representativo de Cristo supone la exaltación del triunfo de este, representación, por tanto, muy adecuada para un contexto funerario.

En línea con la sencillez de ejecución del crismón, la escritura es una capital clásica de ejecución natural con rasgos claramente cursivizados. Conserva restos de líneas guía, lo cual testimonia la preparación previa al trazado de la escritura, mostrando un trabajo muy propio de los talleres epigráficos, tanto en la Hispania romana, como posteriormente durante el dominio de los monarcas visigodos..$^{18}$ Esas líneas guía acotaron el espacio escriptorio mediante las llamadas líneas maestras y facilitaron el trazado de los renglones a través de las líneas rectrices, cuyos restos, aún visibles en la inscripción, acreditan un grabado arañando la superficie previamente pulimentada de la piedra mediante una punta seca. Las proporciones de ese recuadro trazado por las líneas maestras reflejan un trabajo más preciso y profesional de lo que a primera vista podríamos deducir, pues se acercan notablemente a la proporción de la sección áurea, 1,618 de esta por 1,653 de la inscripción, uso de proporciones

Expediente 1942/13.

16 Eusebio de Cesarea relata la legendaria visión del signo de la cruz en los cielos que tuvo Constantino como premonición de su victoria sobre Majencio en la batalla del Puente Milvio; posteriormente el Emperador vio a Cristo en sueños con el signo que había aparecido en el cielo y este le ordenó que fabricara una imitación del signo observado y se sirviera de él como bastión en las batallas (Eus. VC 1.27-32).. El mismo suceso es narrado, con alguna modificación, por Lactancio, quien expone que Constantino en sueños recibió la indicación de grabar en los escudos el signo celeste de Dios, lo que hizo girando la letra X con su extremidad superior curvada (Lact. Mort. Pers. 44). Un sucinto análisis de las diversas formas de representar el nombre de Cristo en Hurtado 2006, 207-211.

17 Término utilizado por Larry W. Hurtado (Hurtado, 2000, 280)

18 Santiago Fernández 2011, 182. 
que no es extraña y que ya ha sido acreditada en otras inscripciones. ${ }^{19}$ El empleo de estas proporciones sirve para dotar de armonía y equilibrio al conjunto. Es un trabajo previo ampliamente utilizado en época tardoantigua. La inscripción cuenta con seis líneas de escritura y, de nuevo, la regularidad es la característica, como muestra el número de letras de cada uno de los renglones, entre las 16 de la que tiene menos, la quinta, y las 19 de las que cuentan con más, la cuarta y la sexta; la primera y la tercera tienen 17 y la segunda 18. Sin embargo, la ordinatio ni mucho menos puede ser calificada de perfecta, como acredita la irregular alineación de algunos renglones, especialmente perceptible en el cuarto, o el hecho de que la última letra de la tercera línea sea trazada sobre la línea maestra.

El tamaño de las letras es irregular. El cuadro siguiente permite apreciar cómo en todas las líneas hay significativas diferencias entre la letra de mayor altura y la más corta, oscilando entre el $29,62 \%$ y el $46,16 \%$, en relación con la medida mayor.

\begin{tabular}{|l|c|c|c|}
\hline & $\begin{array}{c}\text { Altura mÁxima } \\
(\mathrm{CM})\end{array}$ & $\begin{array}{c}\text { Altura mínima } \\
(\mathrm{cm})\end{array}$ & Diferencia \\
\hline lín. 1 & 3,4 & 2,4 & $1(29,41 \%)$ \\
\hline lín. 2 & 3,1 & 1,7 & $1,4(46,16 \%)$ \\
\hline lín. 3 & 3 & 1,9 & $1,1(36,66 \%)$ \\
\hline lín. 4 & 3 & 1,8 & $1,2(40 \%)$ \\
\hline lín. 5 & 3 & 2,1 & $0,9(30 \%)$ \\
\hline lín. 6 & 2,7 & 1,9 & $0,8(29,62 \%)$ \\
\hline
\end{tabular}

Similar es el panorama que nos ofrece el análisis de la relación modular, que hemos estudiado letra por letra para una mayor precisión:

\begin{tabular}{|l|c|c|c|c|c|}
\hline & $\begin{array}{c}\text { NÚMERO DE } \\
\text { LETRAS }\end{array}$ & $\begin{array}{c}\text { RM } \\
\text { MÁXIMA }\end{array}$ & $\begin{array}{c}\text { RM } \\
\text { MínIMA }\end{array}$ & DifERENCIA & $\begin{array}{c}\text { RM } \\
\text { MEDIA }\end{array}$ \\
\hline A & 4 & 1,65 & 1,39 & $0,26(15,75 \%)$ & 1,58 \\
\hline B & 3 & 2,02 & 1,62 & $0,4(19,8 \%)$ & 1,84 \\
\hline C & 4 & 3,01 & 1,53 & $1,48(49,16 \%)$ & 2,19 \\
\hline D & 2 & 1,57 & 1,31 & $0,26(16,56 \%)$ & 1,44 \\
\hline E & 5 & 2,23 & 1,57 & $0,66(29,59 \%)$ & 1,90 \\
\hline F & 3 & 2,16 & 1,78 & $0,38(17,59)$ & 1,93 \\
\hline L & 4 & 2,22 & 1,36 & $0,86(38,73 \%)$ & 1,61 \\
\hline M & 3 & 1,07 & 0,77 & $0,3(28,03 \%)$ & 0,95 \\
\hline N & 10 & 2,08 & 1,19 & $0,89(42,78 \%)$ & 1,62 \\
\hline O & 4 & 1,20 & 1,10 & $0,10(8,30 \%)$ & 1,15 \\
\hline P & 1 & & & & 2,02 \\
\hline R & 3 & 1,95 & 1,26 & $0,69(35,38 \%)$ & 1,56 \\
\hline S & 8 & 2,51 & 2,04 & $0,47(18,72 \%)$ & 2,24 \\
\hline
\end{tabular}

19 Santiago Fernández 2011, 180-181. 


\begin{tabular}{|l|l|l|l|l|l|}
\hline $\mathrm{T}$ & 5 & 1,69 & 1,25 & $0,44(26,03 \%)$ & 1,47 \\
\hline $\mathrm{V}$ & 4 & 1,35 & 1,15 & $0,2(14,81 \%)$ & 1,28 \\
\hline $\mathrm{X}$ & 1 & & & & 0,93 \\
\hline
\end{tabular}

Observamos cómo las oscilaciones son en algunos casos notables. La más regular es la $\mathrm{O}$, con una diferencia del $8,30 \%$, en proporción con la relación modular más elevada, mientras que la letra que ofrece una mayor variabilidad es la $\mathrm{C}$, con un $49,16 \%$, si bien hay otras letras con una variabilidad muy notable como la L, la N o la $\mathrm{R}$, con valores próximos al 40\%. El caso de la $\mathrm{O}$ es aislado, pues todas las demás letras se encuentran en tasas superiores al 15\%.

La relación modular media, obtenida mediante el método propuesto por García Lobo, ${ }^{20}$ es 1,54 , lo cual se adapta perfectamente a lo observado en inscripciones de los siglos IV y $\mathrm{V}$ y se aproxima bastante a la media resultante del análisis individualizado de las letras, 1,61. En este caso el único renglón que se aparta de la media es el primero, puesto que ofrece una relación modular de 1,95, mientras que todos los demás están próximos al 1,5.

La escritura es una capital clásica con fuertes influencias cursivas, como se aprecia en el trazado de la F, con su trazo superior con tendencia ascendente y sobrepasando por la izquierda la unión con el trazo vertical. También en la L, con su segundo trazo hacia abajo cayendo por debajo de la caja de escritura. O la A, con su segundo trazo iniciándose antes de la unión con el primero y ligeramente curvo en ese inicio, rasgo que también observamos en la M. La ejecución de esta letra suele ser uniendo los trazos segundo y tercero en la parte inferior de ambos, apoyando el vértice sobre la línea de escritura; sin embargo, en una de las ocasiones, en la $\mathrm{M}$ del nombre de la difunta, el tercer trazo se apoya sobre la parte central del segundo, particularidad que se explica por la cursividad de la ejecución, si bien no es frecuente; se aprecia en otra inscripción, procedente de Tarragona, ${ }^{21}$ un epitafio en hexámetros que Géza Alföldy dató en el siglo IV, siendo uno de los criterios precisamente el de la escritura. El resto de letras no tienen ninguna otra particularidad digna de ser reseñada, más allá de la naturalidad de ejecución propia de la época.

El análisis diplomático de la inscripción nos ofrece una estructura bastante habitual en los epitaphia sepulchralia cristianos, principalmente en la Bética y en la Lusitania, con una intitulación, acompañada de fórmula de edad, notificación, indicando el fallecimiento o la deposición del cuerpo, y data, introducida por el verbo notificativo, como suele suceder en los epitafios cristianos, si bien carece de la indicación del año, lo cual puede relacionarse con su antigüedad.

Comencemos por el estudio de la intitulación. El nombre Firmana está testimoniado en varias inscripciones romanas, tanto en su forma masculina, como femenina, si bien entre los epitafios cristianos esta inscripción es el único caso conocido. Claramente es de ascendencia romana, como es habitual; de hecho, en la Bética el 95\% de la población era de origen romano, de acuerdo a los análisis antroponímicos realizados. ${ }^{22}$ Además de indicar su condición de niña, se une al nombre una poco usual fórmula de tono afectuoso, anima dulcis. Únicamente ha sido

García Lobo 1999, 153.

Alföldy 1975, 442-443, n 1004.

García de Castro 1995-1996. 
constatada en esta inscripción y en otras dos, una procedente de Carteia (Cádiz), el epitafio de Aurelio Félix, ${ }^{23}$ datado por Javier del Hoyo en los siglos IV-V, ${ }^{24}$ y la otra el epitafio de Licinia, ${ }^{25}$ de Cazalla de la Sierra (Sevilla), correspondiente al 485, como Firmana, una niña, si bien en este caso de 11 años.

El apelativo dulcis sirve para componer una fórmula de afecto hacia la difunta, tal como suele ser relativamente frecuente en los epígrafes cristianos desde comienzos del siglo IV. Este término, o su superlativo, está bien documentado tanto en la epigrafía pagana como en la cristiana; referido a personas y sentimientos, pretende incidir en la afabilidad y el cariño;26 por ello es aplicado con cierta frecuencia a las mujeres, generalmente a las esposas jóvenes y, como en el caso que aquí nos ocupa, a las hijas y niñas. El término dulcissima es bien conocido en la poesía epigráfica clásica para expresar los afectos conyugales. ${ }^{27}$ La mayor parte de las inscripciones que lo utilizan corresponden a los siglos IV o V, salvo alguna excepción posterior. La construcción anima dulcis (o dulcis anima) había sido ya utilizada en epitafios paganos y se mantiene entre los cristianos como expresión de afecto, siendo además muy habitual, como prueban los más de 160 epígrafes que la portan en Roma.

La fórmula de edad es bastante típica, introducida por el verbo vivere en su forma de perfecto, vixit, y constatando los años y los meses, como suele suceder cuando los fallecidos son jóvenes y, especialmente, niños, en particular en los epitafios más antiguos. Lo novedoso de esta inscripción es el empleo del término bonis después del verbo, conformando el formulismo vixit in bonis annis. Es la única inscripción hispana conocida que utiliza esta expresión, que tampoco hemos localizado en otros lugares.

Singular es el verbo notificativo, recollecta est. El término recollecta es empleado en dos inscripciones paganas del norte de África, si bien, bajo mi punto de vista, con un sentido muy diferente, por lo que no serían comparables. Es seguido por el alargamiento in pace, lo cual sirve para cristianizar la fórmula, que creo debemos interpretar como una expresión similar a la mucho más difundida receptus, - $a$ in pace. Tanto en la Bética como en la Lusitania la mención in pace aparece en los primeros epitafios unido al participio receptus, $-a,{ }^{28}$ pero precisamente en vista de este epitafio esa idea debe ser matizada, pues sin duda el de Firmana es de los más antiguos. Es una fórmula que sirve para remarcar el proceso de transición espiritual que conduce a la salvación. ${ }^{29}$

En la data, es importante citar que se menciona el día concreto de la semana, pues no es algo habitual. De hecho, en la Península únicamente conocemos otras cinco inscripciones. ${ }^{30}$ Como suele ser frecuente se prefiere la mención clásica, dies Saturni, a la propiamente cristiana, dies sabbati, pese a las recomendaciones de los Padres de la Iglesia de la conveniencia de emplear los apelativos cristianos en lugar de los de carácter astrológico para referirse a los días de la semana.

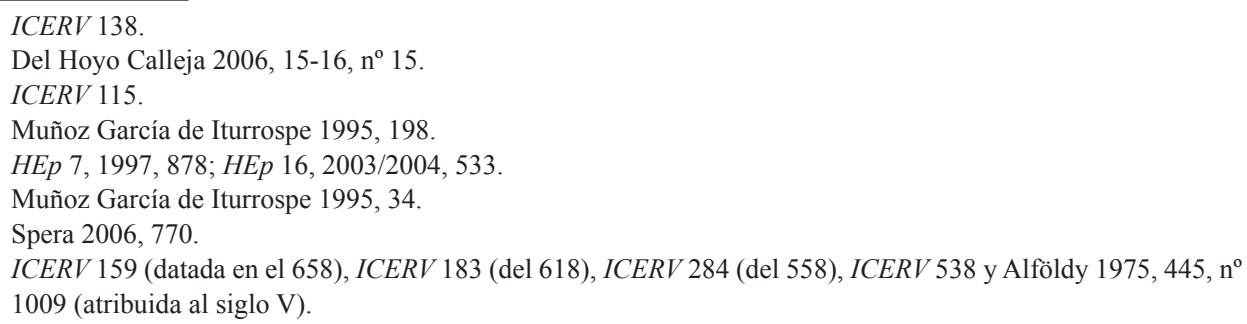


En conjunto, podemos observar que varios formulismos empleados tienen una clara raigambre clásica. Ni siquiera el verbo notificativo, que suele ser un claro exponente del carácter y la fe cristiana, en este caso es inequívocamente cristiano, salvo por ir unido a la expresión in pace, que generalmente tiene la intención de reforzar la expresión del augurio o la certeza del reposo alcanzado por el difunto o de haber alcanzado una condición de beatitud. ${ }^{31}$ Es una expresión que incide en la paz del difunto en el momento de la muerte, en su paz con Dios y con la Iglesia, así como en la paz del sepulcro obtenida por los bienaventurados. ${ }^{32}$

La inscripción ha sido datada por los editores anteriores de manera genérica en los siglos IV o V. Como antes cité la mención al día concreto de la semana nos permite situarla en unos años determinados, todos aquellos en los que el día séptimo de las kalendas de febrero fue sábado. El abanico ofrecido es demasiado amplio como para que esto nos permita una datación aproximada. El primer año es el 318, seguido del 329 , lo cual evidentemente es demasiado antiguo como para ser aceptable como cronología de la inscripción. Después nos vamos al 346, 352, 357, 363, 374, 380, $385,391,402,413,436,441,447,458,464,469,475,486,492$ y 497, pues los años correspondientes a los siglos VI y VII los he desestimado.

El crismón puede ser utilizado como un elemento de datación. Como antes he señalado, se trata de un crismón constantiniano, sin el alfa y omega pendientes. Floriano Cumbreño estableció un modelo general de evolución de los crismones, indicando que en las inscripciones paleocristianas subsiste el crismón constantiniano hasta el final del siglo IV. A finales de dicho siglo se le incorpora un trazo horizontal o se simplifica transformándose en una $\mathrm{P}$ crucífera, más conocida como cruz monogramática. Posteriormente, según el citado autor, a los modelos establecidos se les irán incorporando las apocalípticas letras griegas alfa y omega..$^{33}$

En general, la evolución descrita se cumple en las inscripciones hispanas tardoantiguas, al menos en lo referente al crismón sin alfa y omega, si bien quizá la cronología de su presencia pudiera retrasarse hasta los primeros años del siglo V. Fijar con mayor precisión la evolución cronológica es complicado, pues precisamente los más antiguos epígrafes que portan el crismón con ausencia de alfa y omega suelen carecer de data explícita. Únicamente he localizado el mirtilense epitafio de Orania, correspondiente al $503,{ }^{34}$ pero es un hecho aislado y un dato enormemente curioso que se suma al resto de particularidades de esta inscripción, que quizá reaprovechó un soporte previamente preparado, por lo que considero no debe ser tenida muy en cuenta para el tema que ahora nos ocupa; de hecho, el resto de epígrafes que portan este signo iconográfico la práctica totalidad de editores coinciden en situarlos en el siglo IV o bien, en algunos casos, en los primeros años del V. De cualquier modo, la evolución no es lineal, pues una inscripción de Tarraco, que ya incorpora el alfa y el omega, está datada en el $393^{35}$ y, por citar algún ejemplo más, el epitafio de Flavius Salutius, antes citado, se atribuye al siglo IV. ${ }^{36}$ Estos testimonios ni mucho menos han de suponer que el empleo del crismón sin las citadas letras griegas hubiese desaparecido en esos años.

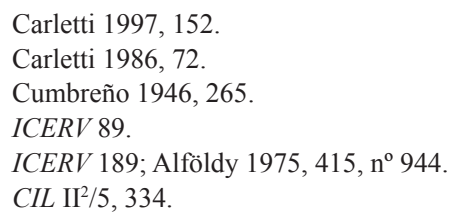


La escritura, una capital clásica cursivizada, sin presencia alguna de la nueva romana y sin el uso de signos gráficos que se desarrollarán en la Hispania visigoda, ratifica la antigüedad de la inscripción y encaja perfectamente con la cronología propuesta de finales del siglo IV o principios del V.

Los formulismos, como antes señalé muy entroncados con el mundo clásico, pueden apuntar al siglo IV, pero sin hacer inviables los primeros años del V. La notificación recollecta est in pace no ha sido testimoniada en ningún otro epígrafe hispano, pero creo que se puede relacionar con las fórmulas alusivas a la depositio, es decir receptus, - a in pace.

En la fórmula de edad se emplea el ablativo de duración, annis, signo de antigüedad, pero también posible en el siglo $\mathrm{V}$ e incluso en épocas posteriores; después del primer cuarto del siglo VI apenas se utiliza, pero el último caso conocido se data en el $609,{ }^{37}$ lo cual demuestra que el uso del ablativo, en lugar del acusativo de extensión, annos, por sí solo no es un criterio fiable de datación. Antes me he referido a la presencia o ausencia del año explícito de datación; se suma a corroborar la cronología a la que me estoy refiriendo, pues es sabido que la primera inscripción datada de manera explícita por la era es la emeritense de Bracarius del $381 .{ }^{38}$ Parece lógico pensar que la que nos ocupa, si es que estuviera datada por la Era, sería posterior; ya señalé anteriormente que los primeros epígrafes datados en la Bética son de la segunda mitad del siglo V.

Como conclusión a todo lo expuesto, creo que el epitafio de Firmana puede ser datado en cualquier de los años de la segunda mitad del siglo IV $(346,352,357,363$, $374,380,385,391)$ o los primeros $(402,413)$ del $V$ en que el día séptimo antes de las kalendas de febrero fue sábado.

En conjunto estamos ante un epitafio que tiene notables puntos de interés, que se derivan no únicamente de su curioso acceso a los fondos del Museo Arqueológico Nacional, sino también por contener determinados formulismos que, aunque conocidos, no son demasiado abundantes en la epigrafía cristiana peninsular, como son el empleo del término infans o, en este caso infas, asociado al nombre, en no más de una decena de inscripciones, la composición anima dulcis, solo constatada en otros dos casos, la expresión vixit in bonis annis, que conforma un unicum en Hispania, o la indicación del día de la semana concreto, que únicamente se ha observado en otros cinco epígrafes de la Península. A todo ello se une lo interesante del análisis externo que, a pesar de la cursivización de la escritura, denota un trabajo profesional, como se deduce de la acotación del espacio escriptorio mediante líneas maestras, del empleo de líneas guía o rectrices y la aproximación del espejo epigráfico a la proporción áurea.

\section{Epitaphium sepulchrale anónimo (Fig. 2)}

Fragmento inferior de una losa sepulcral de caliza, con unas dimensiones de $36 \mathrm{~cm}$ de alto, $18 \mathrm{~cm}$ de ancho y grosor de $5,5 \mathrm{~cm}$. Falta la parte superior y la parte izquierda

\section{ICERV 146.}

38 ICERV 18. Navascués expresó sus dudas acerca de la fiabilidad del texto transmitido por esta inscripción desaparecida, que sería el testimonio más antiguo conocido del uso de la Era Hispánica (Navascués 2019, 779780); el siguiente en antigüedad es el epitafio de Octavia (ICERV 24), del 442. 
de la inscripción. Lo conservado son seis renglones, pero al menos faltan otros tres. Entró en el Museo por donación realizada por Juan Rodríguez Mora en 1960. Nº. inv. 1960/47/24.

\section{RECESSIT \\ [IN] PACE SUB \\ [D]IE DECIMO \\ [K]ALENDAS \\ SPPTEMBRIS \\ [ER]A DCLXXXIII}

- - - - - - / recessit / [in] pace sub $\beta$ [d] ie decimo / [k]alendas / septembris /o [er]a DCLXXXIII.

... descansó en paz el día décimo antes de las kalendas de septiembre (23 de agosto) del año de la era de 683 (645 d.C.).

Bibliografía: Inédito

La restitución del fragmento que falta en la parte superior es inviable. Probablemente contuvo el nombre del fallecido, quizá acompañado del apelativo famulus/a, y de la fórmula de edad con el númeral y annos. Esto es lo más probable dado el repetitivo formulario que suelen tener los epitafios en esta época, pero no es posible aventurar nada más.

La inscripción fue donada al Museo por el comerciante de antigüedades cordobés Juan Rodríguez Mora, según acredita el expediente del archivo del MAN. ${ }^{39}$ El 23 de junio de 1960 el entonces director del Museo, Joaquín Ma de Navascués, acusó recibo de la carta a él remitida por Rodríguez Mora en la que le anunciaba el envío de dos cajas de antigüedades y una piedra labrada. La intención del envío no era la donación, sino la venta, como explícitamente indica Rodríguez Mora en su carta, "le remito unas piedras, para que cuando saquen Vds. los otros que hay ahí, formen un lote de todo cuyo precion (sic) lo dejo a su eleción (sic)"; en el mismo sentido está la contestación de Navascués, "esperamos sus piedras para hacer la propuesta al Ministerio sobre la que ya llegaremos a un acuerdo".

Ese intento de venta se transforma en donación unos meses después. El 26 de julio del mismo año Rodríguez Mora escribe a Navascués para solicitarle que acepte la donación de los dos últimos cajones que había remitido, entre los cuales estaba la inscripción objeto de estudio, así como todo lo que tenía depositado en el Museo desde tiempo atrás. El motivo de ello era el inicio de un largo viaje por América.

En el expediente de ingreso, la inscripción fue inicialmente calificada como "epitafio mozárabe", algo realmente sorprendente dada la constancia de la fecha. Esa clasificación fue posteriormente rectificada de manera correcta, indicando "epitafio visigodo".

El expediente no ofrece ningún tipo de estudio ni transcripción, como sí hace, por ejemplo con una inscripción romana. Simplemente se indica de manera genérica, en referencia al conjunto de piezas, que su lugar de procedencia "probablemente sea

39 Expediente 1960/47/24. 
Córdoba y su provincia", hecho que cuadra perfectamente con el formulario utilizado, especialmente en lo que se refiere a la utilización del verbo notificativo recessit, propio de la Bética en estos años.

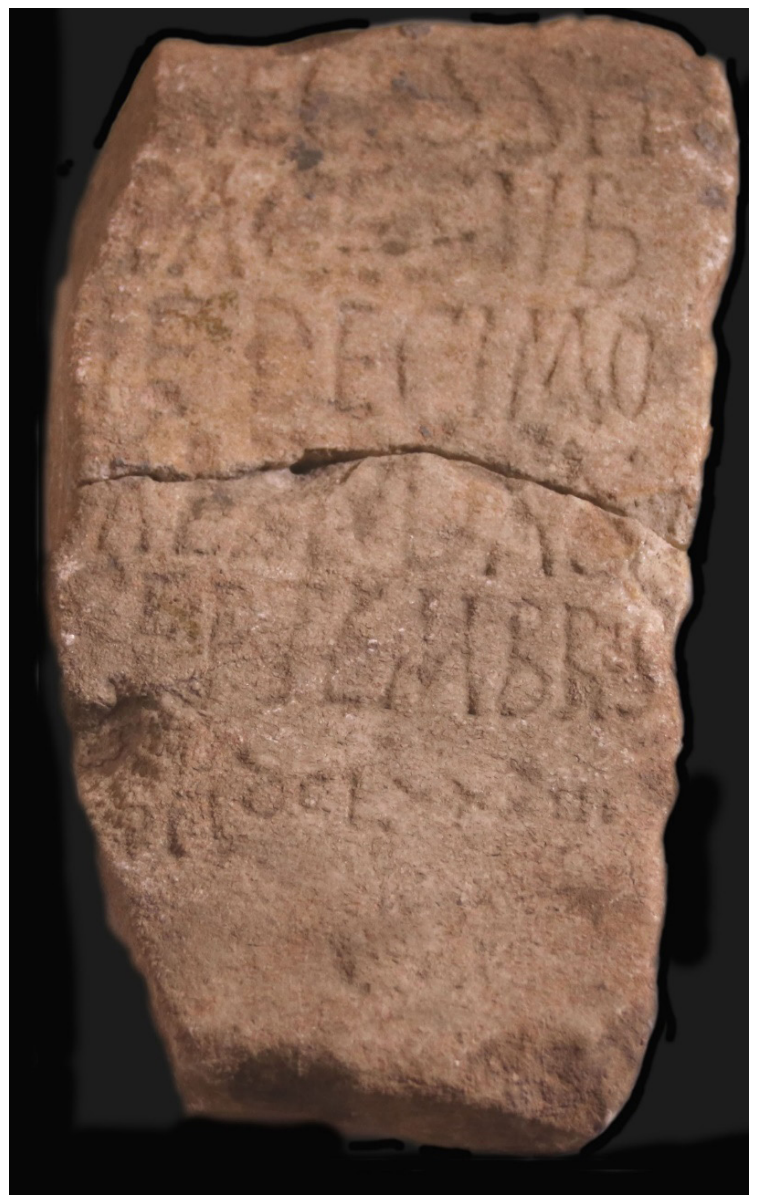

Fig. 2: Epitaphium sepulchrale cordobés anónimo (Fotografía de Elisabeth Menor Natal).

La escritura del epígrafe es la típica capital clásica propia del período, esto es, con una ejecución espontánea y reflejando la influencia de las nuevas formas de la escritura romana y de la cursiva. Asimismo, la data está cursivizada, según suele ser habitual en las inscripciones del período visigodo. Ofrezco a continuación dos cuadros con altura de las letras y con su relación modular, para un más minucioso análisis del epígrafe, en los casos en los que ha sido posible, dado el deterioro del fragmento y excluyendo las grafías de la data por ser cursivas.

\begin{tabular}{|l|c|c|c|}
\hline & $\begin{array}{c}\text { Altura máxima } \\
\text { (сM) }\end{array}$ & $\begin{array}{c}\text { Altura mínima } \\
\text { (сM) }\end{array}$ & Diferencia \\
\hline lín. 1 & 2,9 & 2,8 & $0,1(3,4 \%)$ \\
\hline lín. 2 & 3,4 & 2,3 & $1,1(32,34 \%)$ \\
\hline lín. 3 & 3,6 & 2,9 & $1,1(19,44 \%)$ \\
\hline
\end{tabular}




\begin{tabular}{|l|c|c|c|}
\hline lín. 4 & 4 & 3,1 & $0,9(22,5 \%)$ \\
\hline lín. 5 & 3,7 & 2,9 & $0,8(21,62 \%)$ \\
\hline
\end{tabular}

\begin{tabular}{|l|c|c|c|c|c|}
\hline & $\begin{array}{c}\text { NúMERO DE } \\
\text { LETRAS }\end{array}$ & $\begin{array}{c}\text { RM } \\
\text { MÁXIMA }\end{array}$ & $\begin{array}{c}\text { RM } \\
\text { MíNIMA }\end{array}$ & DifERENCIA & $\begin{array}{c}\text { RM } \\
\text { MEDIA }\end{array}$ \\
\hline $\mathrm{A}$ & 2 & 1,91 & 1,43 & $0,48(25,13 \%)$ & 1,67 \\
\hline $\mathrm{B}$ & 1 & & & & 1,83 \\
\hline $\mathrm{C}$ & 3 & 2,29 & 1,04 & $1,25(54,58 \%)$ & 1,78 \\
\hline $\mathrm{D}$ & 2 & 2 & 1,9 & $0,1(5 \%)$ & 1,95 \\
\hline $\mathrm{E}$ & 3 & 2,46 & 2 & $0,46(18,69 \%)$ & 2,42 \\
\hline $\mathrm{M}$ & 2 & 1,38 & 1,29 & $0,09(6,5 \%)$ & 1,33 \\
\hline $\mathrm{O}$ & 1 & & & & 2,41 \\
\hline $\mathrm{P}$ & 2 & 2,46 & 2,46 & & 2,46 \\
\hline $\mathrm{R}$ & 1 & & & & 3,1 \\
\hline $\mathrm{S}$ & 5 & 2,41 & 1,23 & $1,18(48,96 \%)$ & 1,69 \\
\hline $\mathrm{T}$ & 1 & & & & 1,88 \\
\hline $\mathrm{V}$ & 1 & & & & 1,85 \\
\hline
\end{tabular}

La relación modular media es 2, notable incremento en relación con la inscripción de Firmana, que puede ser relacionado con la cronología más avanzada de este epitafio anónimo, si bien es demasiado aventurado extraer conclusiones al respecto, dado el escaso número de letras analizado por lo fragmentario de su conservación. De hecho, en un análisis que he efectuado con 15 inscripciones situadas entre los siglos V y VII el resultado se aproxima al 1,6, sin que se aprecien significativas diferencias entre los ejemplares procedentes de las distintas centurias.

Dentro de esta caracterización general, hay algunas grafías que merecen un comentario adicional. Es el caso de la U uncial, letra que encontramos en las inscripciones tardoantiguas desde época temprana, en concreto desde finales del siglo IV o principios del V, según atestigua, por ejemplo, el cordobés epitafio de Victoria ${ }^{40}$ si bien es una forma gráfica más propia de los últimos tiempos del dominio visigodo en la Península. En el ámbito cordobés, del que procede nuestra inscripción, la encontramos en los epitafios de Basilia $^{41}$ (650), Columba ${ }^{42}$ (664) y Anerius ${ }^{43}$ (682), lo cual, unido al fragmento aquí analizado, muestra que es una grafía propia de la segunda mitad del siglo VII. Se trata de una letra de amplia proyección posterior, que ha sido presentada como una de las formas características de la escritura visigótica epigráfica a partir del siglo $\mathrm{X},{ }^{44}$ si bien en los siglos IX y X tiene escasa representación, a la luz de los testimonios procedentes de Asturias, ${ }^{45}$ sin duda la región mejor conocida desde el punto de vista epigráfico en los siglos altomedievales.

\footnotetext{
CIL II ${ }^{2} / 7,658$.

CIL II $\mathrm{I}^{2} / 7,779$.

CIL $\mathrm{II}^{2} / 7,705$.

CIL II ${ }^{2} / 7,647$.

García Lobo 1999, 158.

45 Ver Diego Santos 1994.
} 
Esa misma cronología es la propia de la B, cuya característica distintiva es tener sus dos arcos separados en su unión con el vertical. En el contexto cordobés hemos observado esa grafía en los antes citados epitafios de Columba y Anerius. Similar cronología para esta letra observó Navascués en Mérida, identificándola en un epígrafe datado en el $648^{46}$ y viéndola como antecedente de la B típica de los epígrafes mozárabes. Efectivamente, son muy numerosos los epígrafes que podemos catalogar como mozárabes que tienen una B con ese trazado característico, especialmente en Córdoba y su provincia, donde la encontramos desde el año 877 hasta el siglo XI. ${ }^{47}$ Además de en las inscripciones del Sur, este tipo de B también lo localizamos en Asturias, en los siglos VIII y IX, ${ }^{48}$ y en los condados catalanes, entre los siglos IX y $\mathrm{XII},{ }^{49}$ en ambos focos en escasas inscripciones.

Lo mismo sucede con la R, cuyo tercer trazo no confluye con el bucle al unirse con el trazo vertical, con similar cronología y proyección hacia las posteriores inscripciones mozárabes, también con especial incidencia en las del foco cordobés. Quizá la presencia de estas letras claramente vinculadas con las mozárabes medievales pueda explicar la equivocada catalogación inicial en el Museo como inscripción mozárabe.

Desde el punto de vista formular la inscripción no merece mayor comentario, pues se adapta en todo a la estructura de los epitafios de la Bética en esta época.

\section{A modo de conclusión}

En este trabajo planteo el análisis epigráfico de dos interesantes inscripciones del período tardoantiguo. La intención ha sido presentar el estudio de dos epígrafes, pero, al mismo tiempo, de dos objetos museográficos, salvados para la Historia y la investigación histórica por el interés por las Antigüedades y por el coleccionismo tan en boga en tiempos pasados. Aquí los he valorado desde el punto de vista de ser dos objetos creados para obtener una comunicación de tipo publicitario, por tanto, partiendo del planteamiento y objetivos iniciales con los que fueron creados. $\mathrm{Al}$ mismo tiempo, he intentado indagar en su propia historia como objetos históricos, sujetos a diversos avatares, que en unas ocasiones determinan su desaparición y en otras su conservación y su integración en una colección museística, en este caso la del Museo Arqueológico Nacional. La historia de los testimonios del pasado forma también parte de la Historia y, por tanto, de nuestro propio pasado.

\section{Referencias bibliográficas}

Alföldy, G. (1975): Die römischen inschriften von Tarraco (=Madrider Forschungen 10), Heidelberg.

\footnotetext{
46 Navascués 2019, 722, 770-771.

47 Ver González Fernández 2016.

48 Dentro de las inscripciones datadas se localiza en el monumentum aedificationis de la iglesia de la Santa Cruz, en Cangas de Onís, del 737 (Diego Santos 1994, 226-227, n²53) y en la invocatio del ara de Santa María del Naranco (Diego Santos 1994, 101-103, no 74).

49 Santiago Fernández 2003, 62, 89.
} 


\section{Carletti, C.}

(1986): Iscrizioni cristiane a Roma. Testimonianze di vita cristiana (secoli III-VII), (=Biblioteca patristica 7), Firenze.

(1997): "Nascita e sviluppo del formulario epigrafico cristiano: prassi e ideologia", [en] I. di Stefano Manzella (ed.), Le iscrizioni dei cristiani in Vaticano. Materiali e contributi scientifici per una mostra epigrafica, Cittá del Vaticano, 143-164.

Cumbreño, F. (1946): Curso general de Paleografía y Paleografía y Diplomática Españolas, Oviedo, 2 vols.

Diego Santos, F. (1994): Inscripciones medievales de Asturias, Oviedo.

García de Castro, F. J. (1995-1996): "La sociedad bética del siglo IV d.C. a través de las fuentes epigráficas”, Mainake 17-18, 193-205.

García Lobo, V. (1999): “La escritura publicitaria en la Península Ibérica, siglos X-XIII”, [en] W. Koch - Ch. Steininger (hrsg.), Inschrift und Material, Inschrift und Buchschrift. Fachtagung für mittelalterliche und neuzeitliche Epigraphik Ingolstadt 1997 (=Abhandlungen. Bayerische Akademie der Wissenschaften. Philosophisch-Historische Klasse 117), München, 51-190.

González Fernández, J. (2016): Inscripciones mozárabes de Andalucía, Sevilla.

Hoyo Calleja, J. del

(2006): "Catálogo de inscripciones latinas de Carteia", [en] L. Roldán Gómez et alii (eds.), Estudio histórico - arqueológico de la ciudad de Carteia (San Roque, Cádiz) 19941999 (=Junta de Andalucía, Consejería de Cultura. Monografías 24), Sevilla, 1-49.

(2016): "Fórmulas singulares en la epigrafía cristiana de la Bética occidental", [en] J. Carbonell Manils - H. Gimeno Pascual (eds.), A Baete ad fluvium Anam. Cultura epigráfica en la Bética Occidental y territorios fronterizos. Homenaje al profesor José Luis Moralejo Álvarez (=UAH. Obras colectivas. Humanidades 54), Alcalá de Henares, 51-65.

Hurtado, L. W.

(2000): "The Earliest Evidence of an Emerging Christian Materia and Visual Culture: The Codex, the Nomina Sacra and the Staurogram", [en] S. G. Wilson - M. Desjardins (eds.), Text and Artifact in the Religions of Mediterranean Antiquity: Essays in Honour of Peter Richardson (=Studies in Christianity and Judaism 9), Ontario, 271-288.

(2006): "The staurogram in early christian manuscripts: the earliest visual reference to the crucified Jesus?", [en] T. J. Kraus - T. Nicklas (eds.), New Testament Manuscripts. Their Text and Their World, Leiden, 207-226.

Kajanto, I. (1978): “The Hereafter in Ancient Christian Epigraphy”, Arctos 12, 27-53.

Muñoz García de Iturrospe, $\mathrm{M}^{\mathrm{a}} \mathrm{T}$. (1995): Tradición formular y literaria en los epitafios latinos de la Hispania cristiana (=Veleia, Anejos 7), Vitoria-Gasteiz.

Navascués, J. Ma (2019): "Los epígrafes cristianos latinos de Mérida”, [en] J. M. Navascués, Obra epigráfica, Madrid, 693-851.

Pérez de Barradas, J.

(1930): “Excavaciones en la colonia de San Pedro Alcántara (Málaga). Memoria acerca de los trabajos realizados en 1915, 1916 y 1929 por la Sociedad Colonia de San Pedro Alcántara", Junta Superior de Excavaciones y Antigüedades 106, 1-18.

(1932): "La basílica paleocristiana de Vega del Mar (San Pedro de Alcántara - Málaga)", Archivo Español de Arte y Arqueología 8/22, 53-72.

Ramírez Sádaba, J. L. - Mateos Cruz, P. (2000): Catálogo de las inscripciones cristianas de Mérida (=Cuadernos Emeritenses 16), Mérida $(=\mathrm{CICMe})$.

Rodríguez Oliva, P. (1984): “La Antigüedad”, [en] Málaga II. Historia, Granada, 419-444.

Santiago Fernández, J. 
(2003): La Epigrafia latina medieval en los condados catalanes (815-circ. 1150), Madrid. (2011): "La impaginatio en las inscripciones visigodas", [en] M M $^{\mathrm{a}}$ E. Martín López - V. García Lobo (coords.), Impaginatio en las inscripciones medievales (=Corpus Inscriptionum Hispaniae Mediaevalium 1), León, 177-194.

Spera, L. (2006): "Depositus in Cristo. Valenze soteriche nella ridefinizione cristiana del mondo funerario", [en] Pagani e cristiani alla ricerca della salvezza (secoli I- III). XXXIV Encontro di studiosi dell'antichità cristiana. Roma 5 - 7 maggio 2005 (=Studia Ephemeridis Augustinianum 96), Roma, 765-777.

Stylow, A. U. (ed.), (1995): Corpus Inscriptionum Latinarum II: Inscriptiones Hispaniae Latinae, ed. altera, pars VII. Conventus Cordubensis, Berlin-New York (=CIL II $\left.{ }^{2} / 7\right)$.

Stylow, A. U. - Atencia Páez, R. - González Fernández, J. - González Román, C. - Pastor Muñoz, M. - Rodríguez Oliva, P. (eds.), (1998): Corpus Inscriptionum Latinarum II: Inscriptiones Hispaniae Latinae, ed. altera, pars $V$. Conventus Astigitanus, Berlin-New York $\left(=C I L \mathrm{II}^{2} / 5\right)$.

Taracena Aguirre, B. (1947): Adquisiciones del Museo Arqueológico Nacional (1940-1945), Madrid.

Teles Elizalde, M. del C. (2009): "La basílica paleocristiana de Vega del Mar”, Gibralfaro. Aula de momentos de la Historia Menéndez y Pelayo 61, 12.

Vives, J. (1969): Inscripciones cristianas de la España Romana y Visigoda, Barcelona $(=I C E R V)$. 